\title{
Dysrégulation génique dans la trisomie 16 de la souris, modèle de la trisomie 21 humaine
}

Les anomalies du nombre de chromosomes - trisomies ou monosomies sont très mal tolérées par les animaux supérieurs et en particulier par l'homme. Très peu de ces aneuploïdies, lorsqu'elles sont complètes, sont compatibles avec une vie prolongée, la seule courante après la naissance chez l'homme étant la trisomie 21, dont la fréquence est de 1 pour 800 naissances. Chacune d'entre elles, par ailleurs, entraîne des altérations bien définies, et les spécialistes peuvent reconnaître, sur l'aspect clinique, l'anomalie chromosomique en cause. On connaît en outre un nombre croissant de syndromes dus à des délétions localisées, montrant qu'un certain nombre de gènes ont leur fonction altérée lorsqu'ils sont à l'état haploïde. Cependant, toute région génique trisomique ou monosomique ne déclenche pas nécessairement de symptômes pathologiques; l'exemple le mieux connu est celui de la trisomie 21: on sait que c'est la duplication d'un segment du bras long, en 21q22.2-22.3 qui provoque l'ensemble des symptômes du mongolisme, et que celle de la plus grande partie du 21 est sans effets $(\mathrm{m} / \mathrm{s}$ $n^{\circ} 10$, vol. 6, p. 1025 et $n^{\circ} 3$, vol. 7, p. 286).

On ignore par quels mécanismes un nombre anormal de gènes normaux altère les fonctions cellulaires. La relation la plus simple entre nombre de gènes et régulation de l'expression est qu'ARNm et protéine soient proportionnels au nombre de gènes qui codent pour eux (effet de dose). Ce concept, simpliste à première vue, prédit que chaque produit d'un chromosome trisomique soit égal à 1,5 fois celui du témoin normal. Ces dosages ont été effectués sur de nombreuses enzymes humaines, et le plus surprenant est que; la relation y a été en effet observée. C'est même de cette façon qu'il y a vingt ans, avant l'entrée en scène de la biologie moléculaire, on a tir de cellules, y compris des globules rouges, de sujets porteurs de diverses aneuploïdies.

L'idée que tous les gènes, d'une trisomie 21 par exemple, soient exprimés à $150 \%$ de leur valeur normale, et que cela suffise à déclencher les anomalies, n'est pas satisfaisante. La teneur en protéines, en enzymes notamment, est rarement si critique. Lorsqu'on observe que, jusqu'à présent, les dosages ont porté sur des enzymes constitutives, "de ménage ", on peut espérer trouver des protéines à régulations moins automatiques. Celles-ci pourraient être des facteurs de croissance, des protéines structurales ou de membrane, et ce, qu'elles soient codées ou non par le chromosome trisomique. Une équipe unissant des chercheurs de San Francisco (CA, USA) et de West Haven (CT,
USA) [1] a choisi le précurseur de la protéine amyloïde ou APP, dont $\mathrm{m} / \mathrm{s}$ a eu souvent l'occasion de parler. Elle est impliquée dans la genèse de la maladie d'Alzheimer, dont les symptômes apparaissent précocement dans la trisomie 21 , et son gène se situe sur le bras long du 21. C'est dans le système nerveux central que l'APP a été le plus étudiée. Mais, bien que certains travaux [2] aient fait état d'une augmentation de son messager dans le cerveau de trois fotus trisomiques, on ne peut songer à une telle étude systématique chez l'homme. Or il existe chez la souris un modèle comparable à la trisomie 21 humaine, sous forme d'une trisomie 16 ( T 16). Bien que le 16 murin soit plus grand que le 21 humain, de larges portions en sont homologues, et des analogies phénotypiques existent entre les deux syndro-

II existe une méthode générale pour produire des trisomies sur les 19 chromosomes de la souris [3]. On part de translocations robertsoniennes (fusion de deux acrocentriques par leur centromère) qui existent dans la nature et sont répertoriées. L'animal donneur porte une double translocation équilibrée, le chromosome visé, et lui seul, étant inclus dans chacune d'elles. La combinaison avec un animal normal donnera des équilibrés, des monosomies et des trisomies. Le schéma montre comment on arrive à une trisomie.

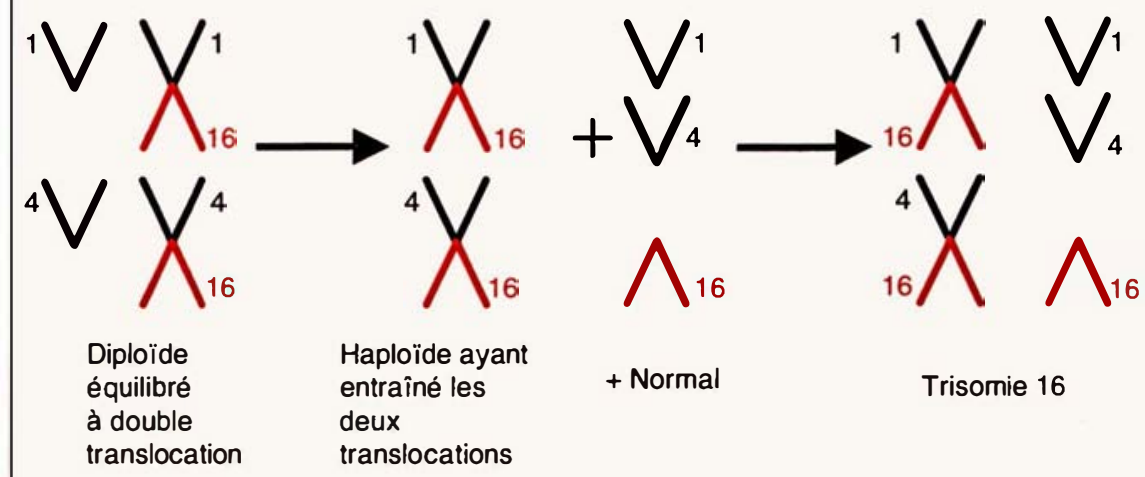

Modèle partant d'une translocation englobant les chromosomes 1 et 4 une fois et 16 deux fois. 


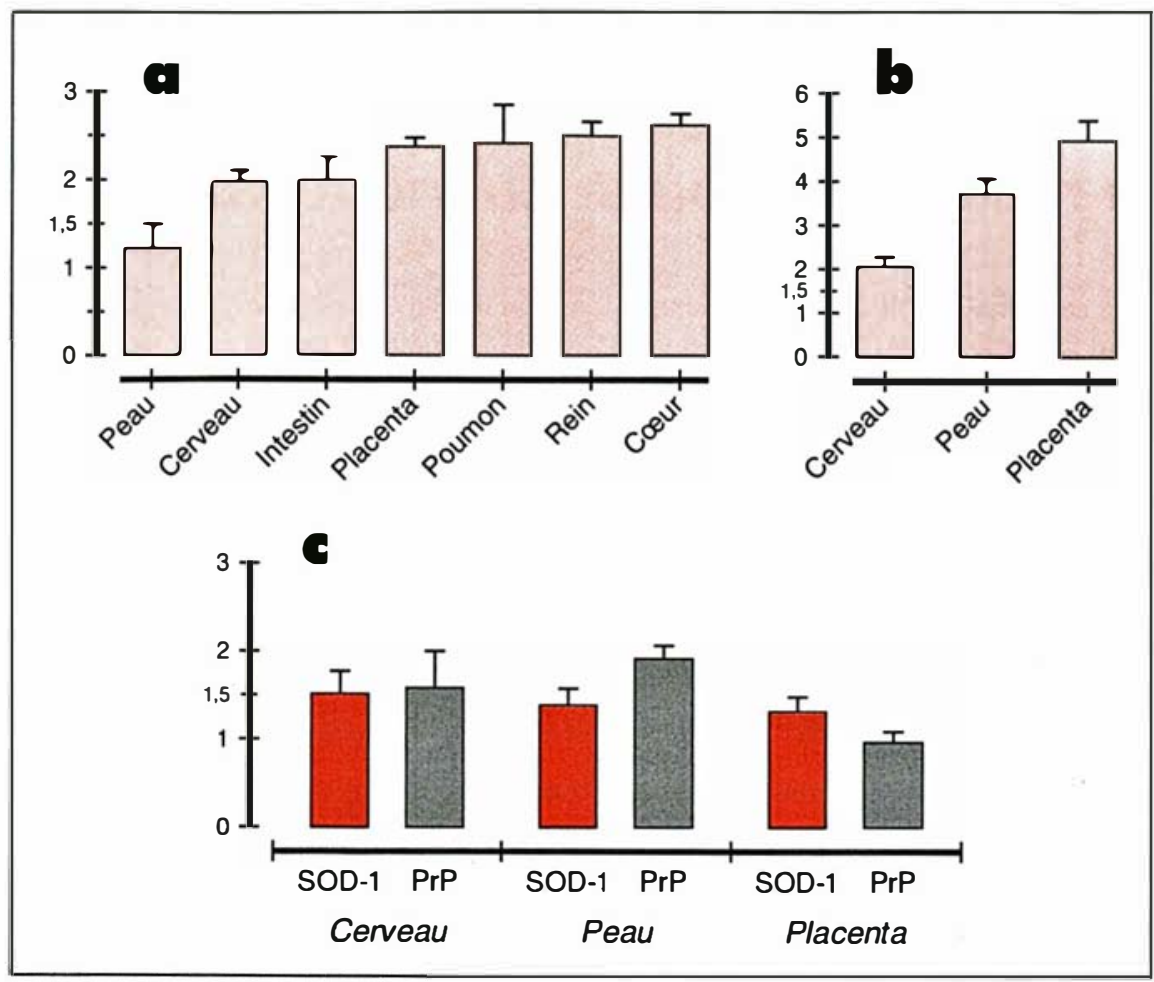

Figure 1. Rapport des taux des ARNm de I'APP, de la SOD et de la PrP chez l'embryon de souris $T 16$ par rapport aux témoins de même portée. $A$ : APP à 15 jours de vie fœetale ; $B$ : APP à 17 jours ; $C$ : SOD et PrP à 17 jours. (D'après [1].)

mes [3] (voir encadre). Les souris T 16 ne survivent pas après la naissance et force est donc de s'adresser aux embryons. On connaît le modèle de développement de l'APP dans le cerveau de la souris. La protéine a plusieurs formes comme chez l'homme, dont trois sont présentes chez l'embryon ; le niveau du messager correspond à celui de la protéine, le transcrit apparaît vers le $15^{\mathrm{e}}$ jour de la vie fotale et augmente jusqu'à la naissance. C'est dans les neurones que le messager est le plus abondant.

Chez les animaux trisomiques (figure 1), le taux de l'ARNm, à 15 et à 17 jours, était significativement différent de 1,5 et atteignait 2, sans changement de localisation cellulaire par rapport au témoin, et sans modification du taux d'ARNm total. Le rapport était de 2,7 dans le cœur et montait jusqu'à 4,9 dans le placenta à 17 jours; dans la peau il passait de 1,2 à 15 jours à 3,5 à 17 jours. On dosa, à titre de " témoin ", la superoxyde dismutase, $m / s n^{\circ} 5$, vol. 8 , mai 92 égal à 1,25-1,30 fois celle des témoins ; le rapport trouvé fut de 3,0 , et de 2,1 pour PrP.

Quelle est la signification de tels résultats ? Un premier problème est celui de leur fiabilité, étant donné la modération des élévations du taux d'expression. Ils ont été statistiquement validés par le test t. Dans les expériences sur l'APP, cinq mesures ont été faites, portant chacune sur huit cerveaux par échantillon de T 16 et de témoins de même portée. Pour PrP furent utilisés trois ou cinq échantillons selon les organes. Dans le cas des adultes, qu'il est sans doute difficile d'obtenir, on n'utilisa que deux chimères, mais un rapport de 3 paraît incompatible avec un effet de dose génique. Des résultats analogues ont été obtenus pour l'APP par une autre équipe [4]. Des augmentations de ce type pourraient exercer un effet sur le développement du système nerveux. Il faudrait toutefois renforcer ces arguments par d'autres. Or, on sait que les essais de transgenèse effectués avec l'APP $(\mathrm{m} / \mathrm{s}$ $n^{\circ} 8$, vol. 7, p. 859) ont été soumis récemment à de sévères critiques $(\mathrm{m} / \mathrm{s}$ $n^{\circ} 4$, vol. 8, p. 400); celles de la PrP $\left(\mathrm{m} / \mathrm{s} n^{\circ} 2\right.$, vol. $7, \quad$ p. 186) attendent confirmation.

Pour tenter d'expliquer les mécanismes conduisant à des régulations non proportionnelles au nombre de gènes, il n'existe que des hypothèses sans argurments convaincants. C'est déjà un progrès notable que d'en avoir montré la possibilité. L'utilisation de modèles animaux aneuploïdes pourra jouer un rôle dans le concert des méthodes contribuant aux progrès dans ce domaine.

J.C. D. notam (présence dans le cervea d'expression ne devrait pas être altéré, en principe, dans la T 16 . Or le rapport trouvé pour l'ARNm de PrP fut de 1,65 dans le cerveau et de 2,0 dans la peau.

On s'efforça ensuite d'explorer le cerveau adulte. La souris T 16 n'est pas viable, mais il est possible d'obtenir des chimères T $16 \leftrightarrow 2 \mathrm{~N}$, dont les tissus peuvent être distingués par des isozymes de la glucose phosphate isomérase ; deux animaux contenant 40-50\% de cellules T 16 ont pu être analysés ; à 6 mois, ils ne présentaient pas d'anomalies histologiques cérébrales. L'ARNm de l'APP aurait dû être

1. Holtzman DM, Bayney RM, Li Y. Dysregulation of gene expression in mouse trisomy 16 , an animal model of Down syndrome. EMBO J 1992 ; 11 : 619-27.

2. Neve RL, Finch EA, Dawes LR. Expression of the Alzheimer anyloid precursor gene transcripts in the human brain. Neuron 1988; 1 : 669-77.

3. Coyle JT, Oster-Granite ML, Reeves RH, Gearhart JD. Down syndrome, Alzheimer's disease and the trisomy 16 mouse. Trends Neurosi $1988 ; 11: 390-4$.

4. Bendotti C. Forloni GL, Morgan RA, et al. Neuroanatomical localization and quantification of amyloid precursor protein mRNA by in situ hybridization in the brains of normal, aneuploid, and lesioned mice. Proc Nall Acad Sci USA 1988 85 : 3628-32.

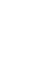

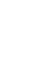

)
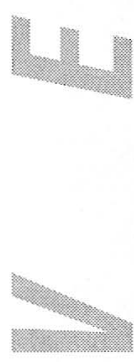